\title{
Lithium-Conducting Self-Assembled Organic Nanotubes
}

Michael J. Strauss, ${ }^{1 \dagger}$ Insu Hwang, ${ }^{2 \dagger}$ Austin M. Evans, ${ }^{1}$ Anusree Natraj, ${ }^{1}$ Xavier Aguilar-Enriquez, ${ }^{1}$ Ioannina Castano, ${ }^{1}$ Emily K. Roesner, ${ }^{1}$ Jang Wook Choi, ${ }^{2 *}$ and William R. Dichtel ${ }^{1 *}$

${ }^{1}$ Department of Chemistry, Northwestern University, 2145 Sheridan Road, Evanston, Illinois 60208, United States ${ }^{2}$ School of Chemical and Biological Engineering and Institute of Chemical Processes, Seoul National University, 1 Gwanak-ro, Gwanak-gu, Seoul 08826, Republic of Korea

† These authors contributed equally to this work

\section{Abstract}

Supramolecular polymers are compelling platforms for the design of stimuli-responsive materials with emergent functions. Here, we report the assembly of an amphiphilic nanotube for Li-ion conduction that exhibits high ionic conductivity, mechanical integrity, electrochemical stability, and solution processability. Imine condensation of a pyridine-containing diamine with a triethylene glycol functionalized isophthalaldehyde yields pore-functionalized macrocycles. Atomic force microscopy, scanning electron microscopy, and in solvo X-ray diffraction reveal that macrocycle protonation under their mild synthetic conditions drives assembly into high-aspect ratio $\left(>10^{3}\right)$ nanotubes with three interior triethylene glycol groups. Electrochemical impedance spectroscopy demonstrates that lithiated nanotubes are efficient $\mathrm{Li}^{+}$conductors, with an activation energy of $0.42 \mathrm{eV}$ and a peak room temperature conductivity of $3.91 \times 10^{-5} \mathrm{~S} \mathrm{~cm}^{-1}$. ${ }^{7} \mathrm{Li} \mathrm{NMR}$ and Raman spectroscopy demonstrate that lithiation occurs exclusively within the nanotube interior and implicates the glycol groups in facilitating efficient $\mathrm{Li}^{+}$ transduction. Linear sweep voltammetry and galvanostatic lithium plating-stripping tests reveal that this nanotube-based electrolyte is stable over a wide potential range and supports long-term cyclability. These findings demonstrate how coupling synthetic design and supramolecular structural control can yield highperformance ionic transporters that are amenable to device relevant fabrication. More broadly, these results demonstrate the technological potential of chemically designed self-assembled nanotubes.

\section{$\mathrm{Li}^{+}$Conducting Pore-Functionalized Nanotubes}

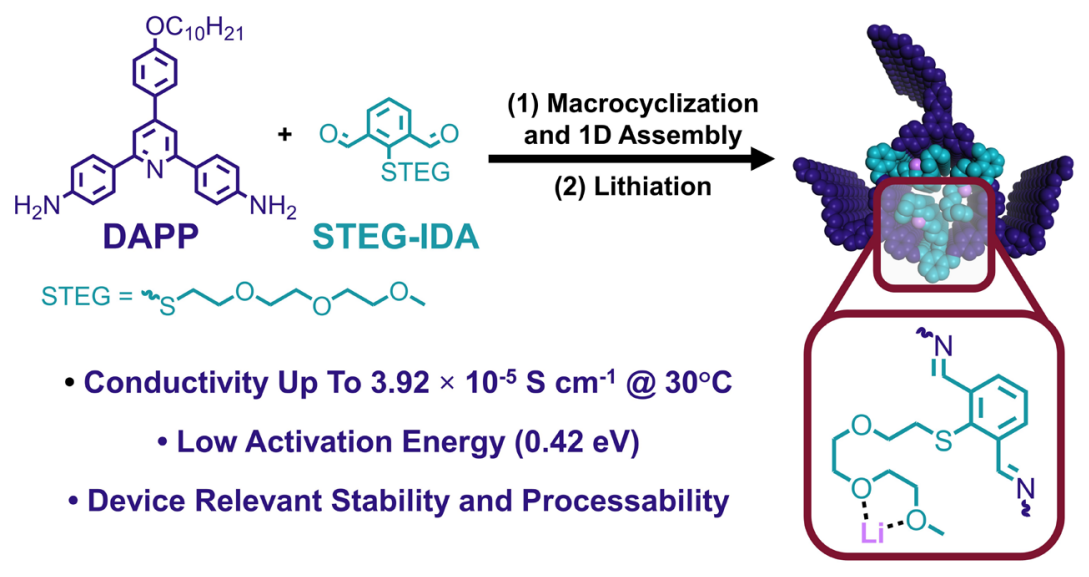




\section{Introduction}

Supramolecular polymers offer stimuli-responsive optical, ${ }^{1-5}$ mechanical, ${ }^{6-9}$ or out-of-equilibrium behaviors that emerge from molecular organization. ${ }^{10-13}$ The ability to leverage these properties will benefit from developing tunable systems whose assemblies are mechanically and chemically robust, yet remain reversible and responsive. Recently, we found that imine-linked macrocycles (ca. $3 \mathrm{~nm}$ diameter) form strong, high-aspect ratio nanotubes upon the protonation of basic groups within their structure. Protonation establishes electrostatic and solvophobic interactions that endow the assemblies with mechanical strengths that rival linear covalent polymers, while still being fully reversible upon deprotonation. ${ }^{14-16}$ This approach was recently generalized and elaborated through the development of a one-pot macrocyclization and 1D assembly strategy that relied on a conserved pyridine-containing diamine monomer. ${ }^{17}$ Condensation with many functional aromatic dialdehydes yielded chemically and structurally diverse macrocycles in high yield, driven by assembly into the corresponding nanotube. This synthetically general approach allows us to systematically tune the properties of the nanotube interior by introducing chemical functionality. Given the charged nature of the nanotube interior, and the opportunity to incorporate groups to facilitate ion transport, we hypothesized that appropriately designed nanotubes might serve as effective ion conductors.

In devices that necessitate efficient ion transport, such as lithium (Li)-ion batteries (LIBs) ${ }^{18-20}$ and fuel cells, ${ }^{21-23}$ designed polymers show promise as safer replacements for liquid-based electrolytes. In LIBs, poly(ethylene oxide) (PEO) is widely used due to its simplistic fabrication and highly competitive ionic conductivity. In an effort to expand the available materials pool beyond PEO, novel covalently-linked polymer systems, such as covalent organic frameworks (COFs), have been explored as ionic conductors. ${ }^{24-}$ ${ }^{26}$ Despite the promising results of these polymer-based electrolytes, balancing the need for highconductivities and mechanical robustness in a processable platform remains challenging in covalently linked systems. ${ }^{27-30}$ In particular, two-dimensional COFs are often prepared as unprocessable, nonuniform particles, such that $\mathrm{Li}^{+}$ion transport in the electrolyte layer and the materials mechanical properties may not be consistent over different cells. ${ }^{24,31}$ Given the solution processability and mechanical robustness of our assembled nanotubes, they are ideally suited for exploration as solid-state Li-ion electrolytes. Synthetic nanotubes offer chemically distinct microenvironments as a handle to tune ionic conductivity within the interior, while also being amenable to exterior chemical modifications that can confer stability. Moreover, because these robust nanotubes undergo acid-responsive assembly, it is conceivable that ionic transport could be manipulated by tuning the extent of macrocycle ordering. Taken together, these 
synthetic nanotubes combine the solution processability and stability of covalent polymers with the welldefined channels of ordered materials. Collectively, these features provide the opportunity to systematically explore optimized ion transduction through coupled molecular and supramolecular design.

Here, we design an amphiphilic system with three triethylene glycol moieties within the nanotube interior (Figure 1). Given the long-range order of the nanotubes, and the propensity of glycols to coordinate $\mathrm{Li}^{+},{ }^{3-}$ 38 we hypothesized that these supramolecular assemblies would be suitable for solid-state Li-ion conduction. Upon lithiating the nanotubes with 1.5 equiv of $\mathrm{Li}^{+}$per macrocycle, a room temperature conductivity of $3.91 \times 10^{-5} \mathrm{~S} \mathrm{~cm}^{-1}$ was realized. By comparing the $\mathrm{Li}^{+}$conduction performance of functionalized nanotubes with a series of control samples, we unambiguously demonstrate that both longrange order and chemical functionality play a critical role in achieving $\mathrm{Li}^{+}$transduction. The well-defined Li-ion transport was beneficially adopted in an emerging post-LIB system, Li-metal batteries, by offering reversible Li plating and stripping over 500 hours of continuous operation. These findings demonstrate that chemically functionalized, solution processable nanotube assemblies can serve as promising solidstate electrolytes. More broadly, these findings reveal that supramolecular assemblies can provide the advantages inherent to synthetically tunable polymeric systems in a solution processable platform.

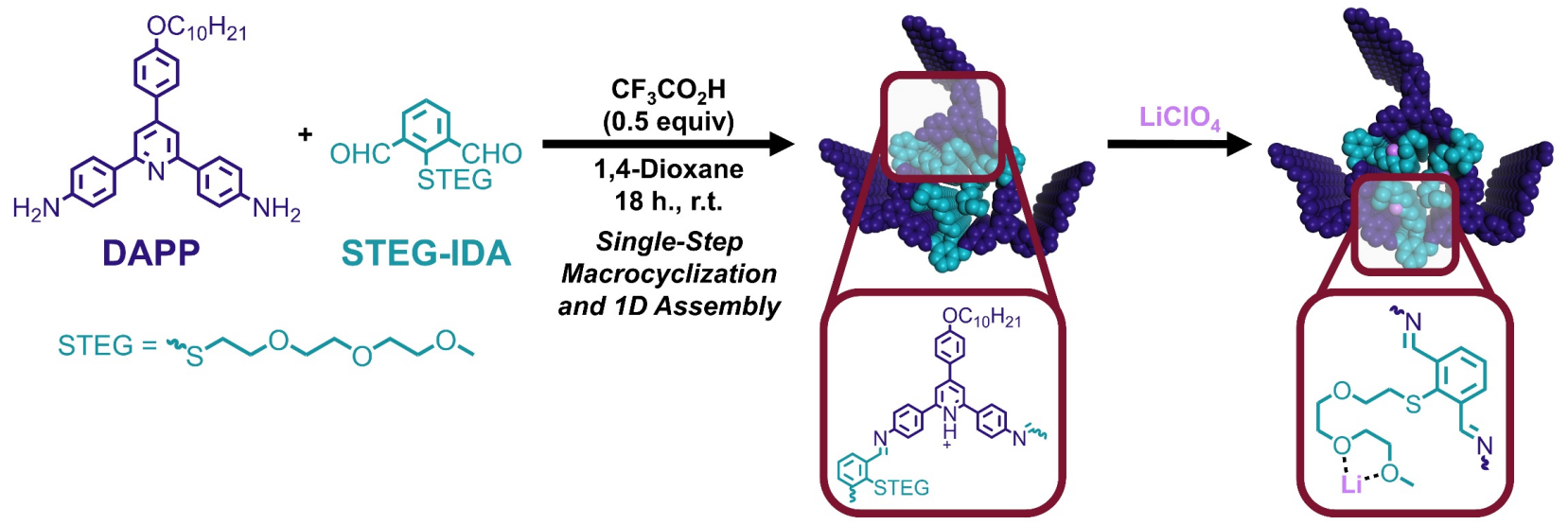

Figure 1. The preparation of nanotubes bearing three interior triethylene glycol groups for emergent Liion conduction.

\section{Results and Discussion}

Amphiphilic macrocycles (STEG-MCs) were synthesized by condensing a 2,4,6-triphenylpyridine-based diamine (DAPP) and 2-(4',7',10'-trioxyundecylsulfanyl)isophthalaldehyde (STEG-IDA) in the presence of $\mathrm{CF}_{3} \mathrm{CO}_{2} \mathrm{H}$ (0.5 equiv per DAPP) in 1,4-dioxane (Figure 2A and Scheme S9). After neutralization, isolation by centrifugation, and washing with $\mathrm{Et}_{2} \mathrm{O}$ and 1,4-dioxane, STEG-MCs were obtained in an isolated yield of $88 \%$. Gel permeation chromatography (GPC) of the macrocycles showed a single narrow elution band $(\nexists=1.04)$, consistent with the formation of a single macrocyclic product (Figure $2 \mathrm{~B}$ and 
S9). Matrix-assisted laser desorption ionization mass spectrometry (MALDI-MS) provided the expected $[\mathrm{M}+\mathrm{H}]^{+},[\mathrm{M}+\mathrm{Na}]^{+}$, and $[\mathrm{M}+\mathrm{K}]^{+}$adducts at $m / \mathrm{z}=2309.80,2331.75$, and 2347.65 respectively (Figure 2C and S10). The macrocycle exhibited a ${ }^{1} \mathrm{H}$ NMR spectrum consisting of well-defined resonances with integrations that are consistent with a symmetric, macrocyclic structure. Resonances corresponding to free aldehyde or amines are not observed (Figures 2D and S11-S13), indicating near complete conversion of the starting materials to the desired macrocycle. Similarly, the ${ }^{13} \mathrm{C}$ NMR spectrum consists of 32 distinct resonances, consistent with the structure of STEG-MC (Figure S14). The high-isolated yield and high purity of the isolated macrocycles highlights the efficient macrocyclization process, which we have previously demonstrated to be driven by an out-of-equilibrium crystallization process that drastically reduces the reactivity of the imines, ${ }^{16,39}$ and enables these materials to be considered for further study and device integration.

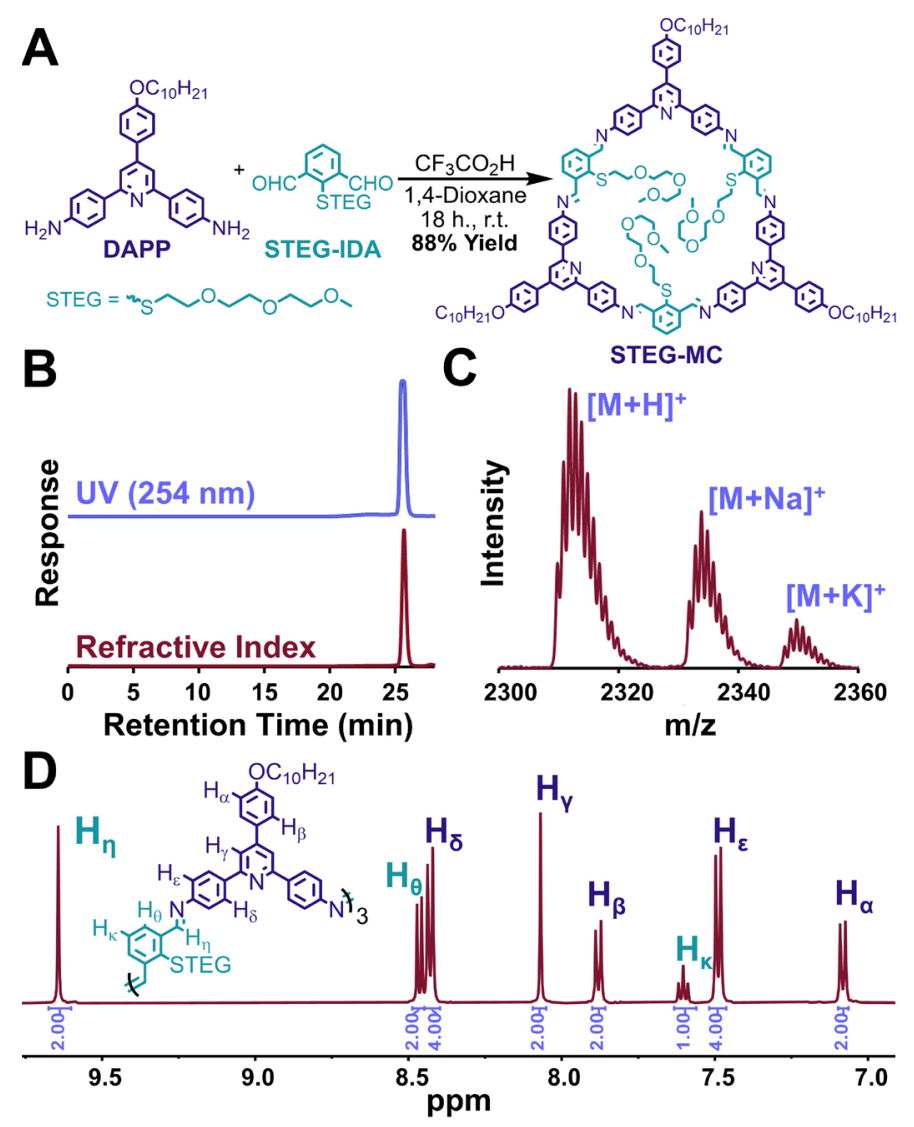

Figure 2. Synthesis and characterization of amphiphilic macrocycles (STEG-MC). (A) Scheme of STEG-MC synthesis. (B) Gel permeation chromatograms of the amphiphilic macrocycles using UV (254 nm, purple) and refractive index (maroon) detectors. (C) Partial MALDI-MS spectrum of STEG-MCs. A full spectrum is available in the supporting information (Figure S10). (D) Partial ${ }^{1} \mathrm{H}$ NMR spectrum highlighting the well-defined resonances within the aromatic region of STEG-MC. A full spectrum and complete assignments are available in the supporting information (Figures S11-S13). 
Protonation of the pyridine moieties within STEG-MC during its synthesis drives assembly into crystalline, high-aspect ratio nanotubes (STEG-NTs), as evaluated by in solvo synchrotron X-ray diffraction (XRD), atomic force microscopy (AFM), and scanning electron microscopy (SEM). After allowing the macrocyclization reaction to run for $18 \mathrm{~h}$, the reaction mixture was placed into a capillary tube and subjected to synchrotron XRD measurements. The assembly of DAPP-based macrocycles is characterized by the emergence of a strong diffraction signal related to the macrocycle size between 0.1 and $0.2 \AA^{-1} \cdot{ }^{17}$ For STEG-NTs, nanotube formation is confirmed by observing this diffraction peak at 0.17 $\AA^{-1}$ (Figure 3B). A second more prominent diffraction feature is observed at $0.2 \AA^{-1}$, which we attribute to increased electron density from the triethylene glycol groups within the nanotube interior, relative to nanotubes that lack such functionality. The experimentally obtained XRD pattern is in good agreement with a Pawley refined pattern corresponding to macrocycle assembly into packed nanotubes with three glycol chains per macrocycle in their interiors (Figures 3B and 3C). AFM and SEM further validated that these nanotubes are formed with significant long-range order. A diluted aliquot of the macrocyclization reaction was drop cast onto a silicon wafer and imaged by AFM. Subsequently, the substrates were sputter coated with $1 \mathrm{~nm}$ of osmium and imaged by SEM. In both cases, high-aspect ratio $\left(>10^{3}\right)$ nanotubes were observed with lengths exceeding $10 \mu \mathrm{m}$ (Figure 3D-F). Taken together, in solvo XRD and direct imaging observations highlight that high-aspect ratio, pore-functionalized nanotubes are efficiently prepared in a single-step from synthetically designed starting materials. 

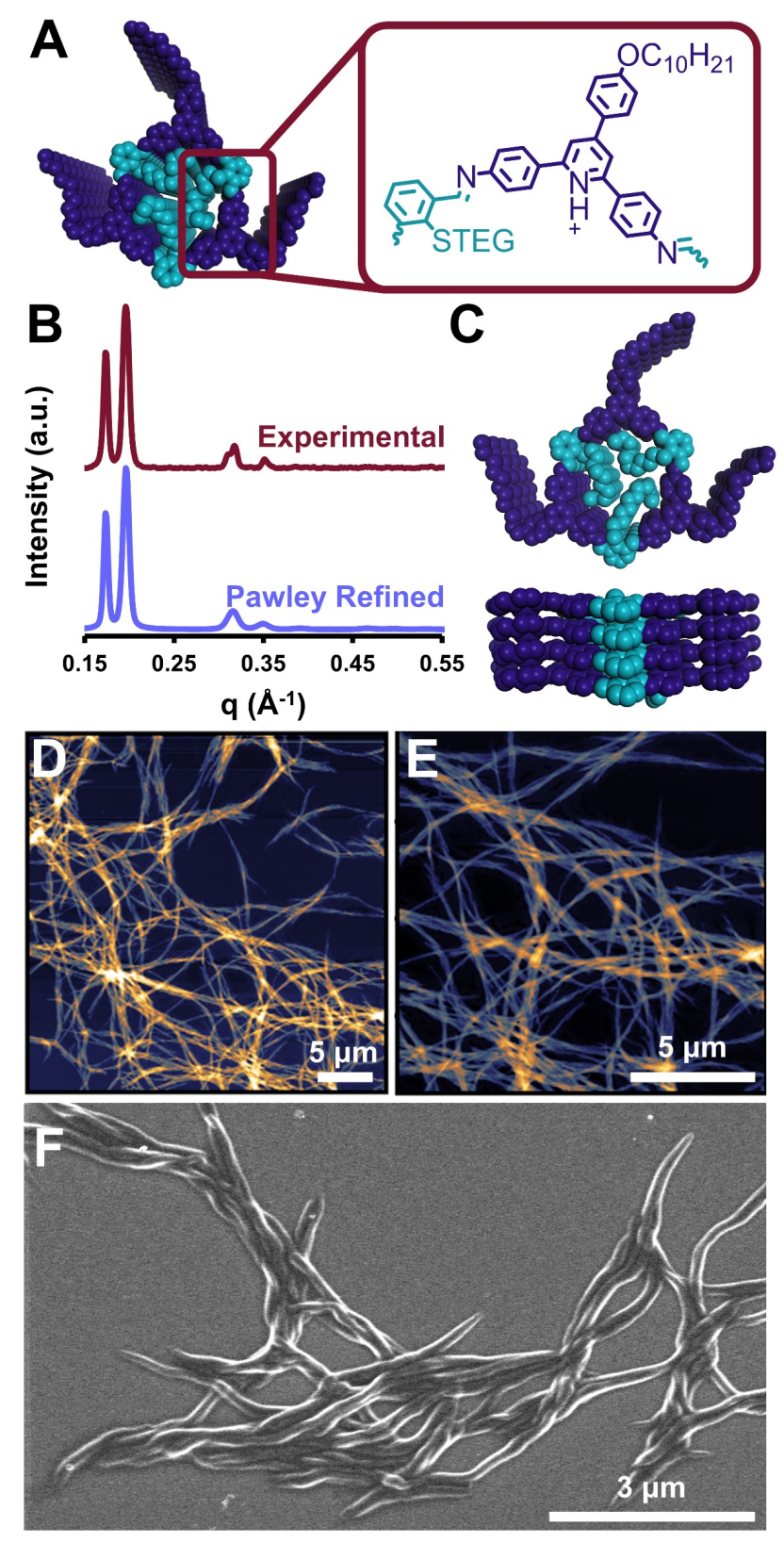

Figure 3. Characterization of STEG-NTs formed during the acid-catalyzed macrocycle synthesis. (A) Schematic of the structure of STEG-NT. (Inset) Chemical structure depicting the protonation of the central pyridine groups that are responsible for macrocycle assembly. (B) Comparison of experimentally obtained XRD patterns of STEG-NTs (top) with the pattern obtained from Pawley refinement (bottom). (C) Structure of the assembly arising from Pawley refinement, validating that the use of 2-functionalized isophthalaldehyde derivatives (STEG-IDA) yield pore functionalized nanotubes. (D and E) AFM images of STEG-NTs. (F) SEM image of STEG-NTs.

The ability to immobilize triethylene glycol residues within the nanotube interior motivated studies into their potential as Li-ion conductors under electrical biasing. STEG-NTs were loaded with varying equivalents of $\mathrm{LiClO}_{4}$ and a limited amount of propylene carbonate as a plasticizer ( $\mathrm{PC}, 10 \mu \mathrm{L} \mathrm{g}^{-1}$ ), pressed into a pellet, and subjected to electrochemical impedance spectroscopy (EIS, Figure 4A and S41). The 
lithium content of the pellets was measured by inductively coupled plasma optical emission spectroscopy (ICP-OES). In all cases, the experimentally obtained $\mathrm{Li}^{+}$content was in good agreement with the $\mathrm{Li}^{+}$feed ratio (Table S1). STEG-NTs were lithiated with 0.5-3.0 equiv $\mathrm{Li}^{+}$per macrocycle, and a maximum conductivity $\left(3.91 \times 10^{-5} \mathrm{~S} \mathrm{~cm}^{-1} @ 30^{\circ} \mathrm{C}\right)$ was observed upon loading STEG-NTs with 1.5 equiv $\mathrm{Li}^{+}$per macrocycle. Compared to bulk poly(ethylene oxide) (PEO), which has a room temperature conductivity on the order of $10^{-8} \mathrm{~S} \mathrm{~cm}^{-1},{ }^{40}$ the high conductivity realized within STEG-NTs highlights the benefit of immobilizing glycol residues within the well-defined, layered nanotube scaffold. Additionally, the observed Li-ion conductivity within STEG-IDA NTs surpasses the room temperature conductivity of many polymers and framework materials designed for $\mathrm{Li}^{+}$conductivity, further demonstrating the promise of assembled macrocycles for this application (Table S2). Deviation of the optimal lithium loading to lower values resulted in decreased conductivity by up to a two orders of magnitude $\left(0.5\right.$ equiv, $3.00 \times 10^{-}$ ${ }^{7} \mathrm{~S} \mathrm{~cm}^{-1} @ 30{ }^{\circ} \mathrm{C}$ ). Higher lithium loadings also showed a deleterious effect on conductivity (3 equiv, 6.09 $\times 10^{-6} \mathrm{~S} \mathrm{~cm}^{-1} @ 30^{\circ} \mathrm{C}$, Figures 4B and 4C). We speculate that appropriately balanced vacant and occupied $\mathrm{Li}^{+}$coordinating sites are realized in samples lithiated with 1.5 equiv of $\mathrm{Li}^{+}$per macrocycle, leading to the observed peak in conductivity. ${ }^{20,41-42}$ This is consistent with previous reports that demonstrate optimal ionic conductivity upon establishing a $[\mathrm{O}]:\left[\mathrm{Li}^{+}\right]$ratio of 6 in triethylene glycol-based electrolytes. ${ }^{43-44}$ Variable temperature EIS on lithiated STEG-NTs (1.5 equiv) demonstrated Arrhenius behavior with an activation energy $\left(E_{\mathrm{a}}\right)$ of $0.42 \mathrm{eV}$ (Figures 4D and 4E), which is significantly lower than that of a framework material with similar functionality $(0.87 \mathrm{eV}) .{ }^{30} \mathrm{We}$ speculate that the lower activation energy realized within STEG-NTs is due to enhanced 1D order as a function of acid-mediated assembly and to the smaller pore size which results in a higher density of layered glycol groups. Taken together, the substantial Li-ion conductivity and low activation energy highlight an efficient conduction process along the channels of STEG-NTs. 

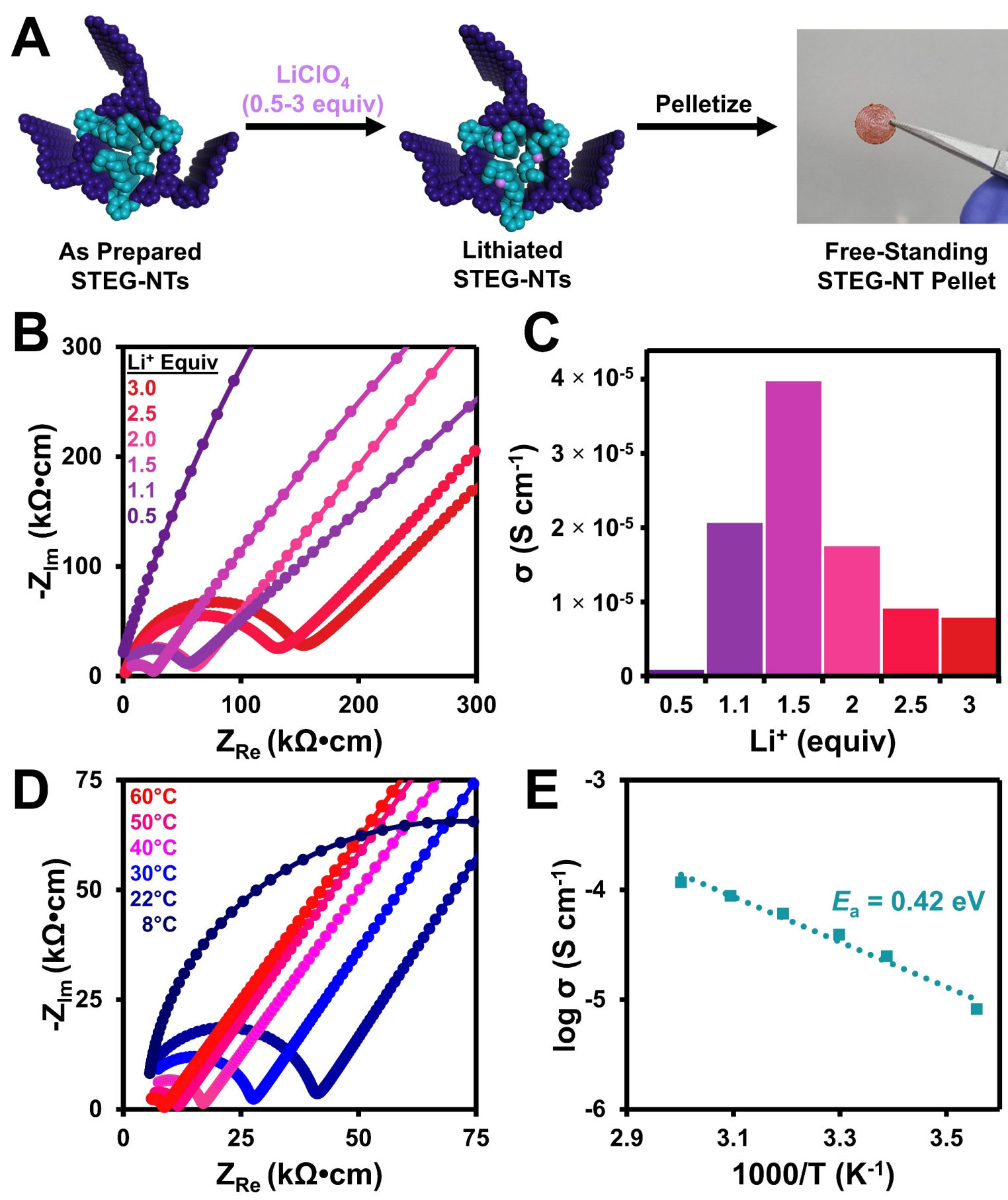

Figure 4. Li-ion conductivity performance of STEG-NTs. (A) Abbreviated schematic of nanotube lithiation and pelletization. A complete workflow diagram for the preparation of the samples for EIS can be found in the supporting information (Figure S41). (B) Nyquist plots obtained from STEG-NTs loaded with varying equivalents of $\mathrm{Li}^{+}$. These spectra were taken at $30^{\circ} \mathrm{C}$. (C) Ionic conductivity as a function of $\mathrm{Li}^{+}$loading, highlighting a peak conductivity at 1.5 equiv $\mathrm{Li}^{+}$. (D) Nyquist plots obtained from variable temperature EIS on STEG-NT pellets. (E) Arrhenius plot of STEG-NT loaded with 1.5 equiv of $\mathrm{Li}^{+}$.

STEG-NTs exhibited chemical and structural stability during the pelletization and measurement process as evident by XRD and ${ }^{1} \mathrm{H}$ NMR spectroscopy obtained after the conductivity measurements were 
performed. After EIS, powder XRD of the pellets revealed a strong diffraction feature at all lithium loadings, indicating that the nanotube structure survived both the pelletization process and the conductivity measurements (Figure S34). Intact, pure STEG-MCs were recovered by dispersing a ground pellet in triethylamine. The insoluble macrocycles were recovered by centrifugation, and their identity and purity was confirmed by ${ }^{1} \mathrm{H}$ NMR spectroscopy (Figure S37). These data highlight the molecular and supramolecular stability of STEG-NTs throughout the cell fabrication and measurement process. Moreover, these findings validate that the molecular scale design and subsequent nanoscale assembly are manifested in these measured devices.

To further investigate if the glycol groups within STEG-NTs play a crucial role in facilitating Li-ion transduction, the effects of nanotube lithiation were investigated through ${ }^{7} \mathrm{Li} \mathrm{NMR}$ and Raman spectroscopy. ${ }^{7} \mathrm{Li}$ NMR spectroscopy demonstrated the ability of the triethylene glycol groups within STEG-NTs to coordinate Li-ions as evident by the nanotubes inducing an upfield shift of the $\mathrm{Li}^{+}$ resonance $(-0.03 \mathrm{ppm})$ relative to a reference sample of $\mathrm{LiCl}(0.50 \mathrm{ppm})$. As the equivalents of $\mathrm{LiCl}$ were increased, the $\mathrm{Li}^{+}$resonance shifted downfield, consistent with the saturation of the coordinating glycol groups (Figure 5A). When compared to lithiated STEG-MCs, lithiated STEG-NTs exhibited a more upfield $\mathrm{Li}^{+}$resonance ( 0.38 and $-0.03 \mathrm{ppm}$, respectively), indicating more robust $\mathrm{Li}^{+}$coordination as a result of layering the glycol groups within the well-defined nanotube scaffold (Figures S31 and S32). ${ }^{32-35}$, ${ }^{45}$ Raman spectroscopy on pellets of STEG-NTs that had been lithiated with 1.5 equiv of $\mathrm{LiClO}_{4}$ further validated that all Li-ions are coordinated by the glycol groups within the nanotube interior. Raman spectroscopy focused on the $\mathrm{Cl}-\mathrm{O}-\mathrm{Cl}$ symmetric stretching frequency of the $\mathrm{ClO}_{4}{ }^{-}$anion between $920-965$ $\mathrm{cm}^{-1}$ provided insight into the solvation state of $\mathrm{Li}^{+}$. The aggregated ions (AGG), contact ion pair (CIP), and free anion have distinct stretching frequencies at 958, 947, and $934 \mathrm{~cm}^{-1}$, respectively (Figures 5B and 5C). ${ }^{46-49}$ Using standard solutions of $\mathrm{LiClO}_{4}$ in $\mathrm{PC}$ it was demonstrated that the free anion is present at low $\mathrm{Li}^{+}$concentrations $\left(1 \mathrm{~mol} \mathrm{~kg}^{-1}\right)$, demonstrating full solvation of the $\mathrm{Li}^{+}$cation by $\mathrm{PC}$. However, as the concentration of $\mathrm{LiClO}_{4}$ was increased, the dominant peak shifted towards the CIP stretching frequency due to the inability of the $\mathrm{PC}$ to fully coordinate the $\mathrm{Li}^{+}$cations at higher concentrations (Figure 5B). When STEG-NTs were lithiated with 1.5 equiv of $\mathrm{LiClO}_{4}$, an intense feature corresponding to the free anion was observed at $933 \mathrm{~cm}^{-1}$, indicating predominant coordination of $\mathrm{Li}^{+}$within the nanotube interior. A small secondary feature corresponding to the CIP was also observed at $947 \mathrm{~cm}^{-1}$, which we attribute to a small amount of $\mathrm{ClO}_{4}{ }^{-}$intercalating into the nanotube interior. The shape of the Raman profile with regards to relative intensities of the free anion and CIP peaks was maintained after adding PC $\left(10 \mu \mathrm{L} \mathrm{g}^{-1}\right)$, indicating the $\mathrm{Li}^{+}$ions remained stably coordinated by the glycol residues within STEG-NTs without 
leaking (Figure 5B). Moreover, this demonstrates that the glycol functionalized interiors of the nanotubes function as genuine $\mathrm{Li}$-ion conductors by providing channels for exclusive $\mathrm{Li}^{+}$transduction. The position

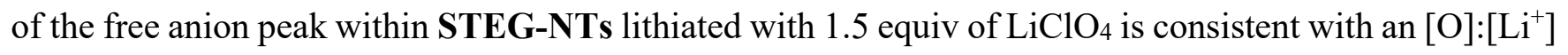
ratio of 6 , as determined by a series of standard solutions of $\mathrm{LiClO}_{4}$ in $\mathrm{PC}$, consistent with the stoichiometric value obtained from ICP-OES (Figure 5D and Table S1). Taken together, ${ }^{7} \mathrm{Li}$ NMR and Raman spectroscopy analyses on lithiated STEG-NTs validate that Li-ions are exclusively coordinated by the glycol groups within the nanotube interior.
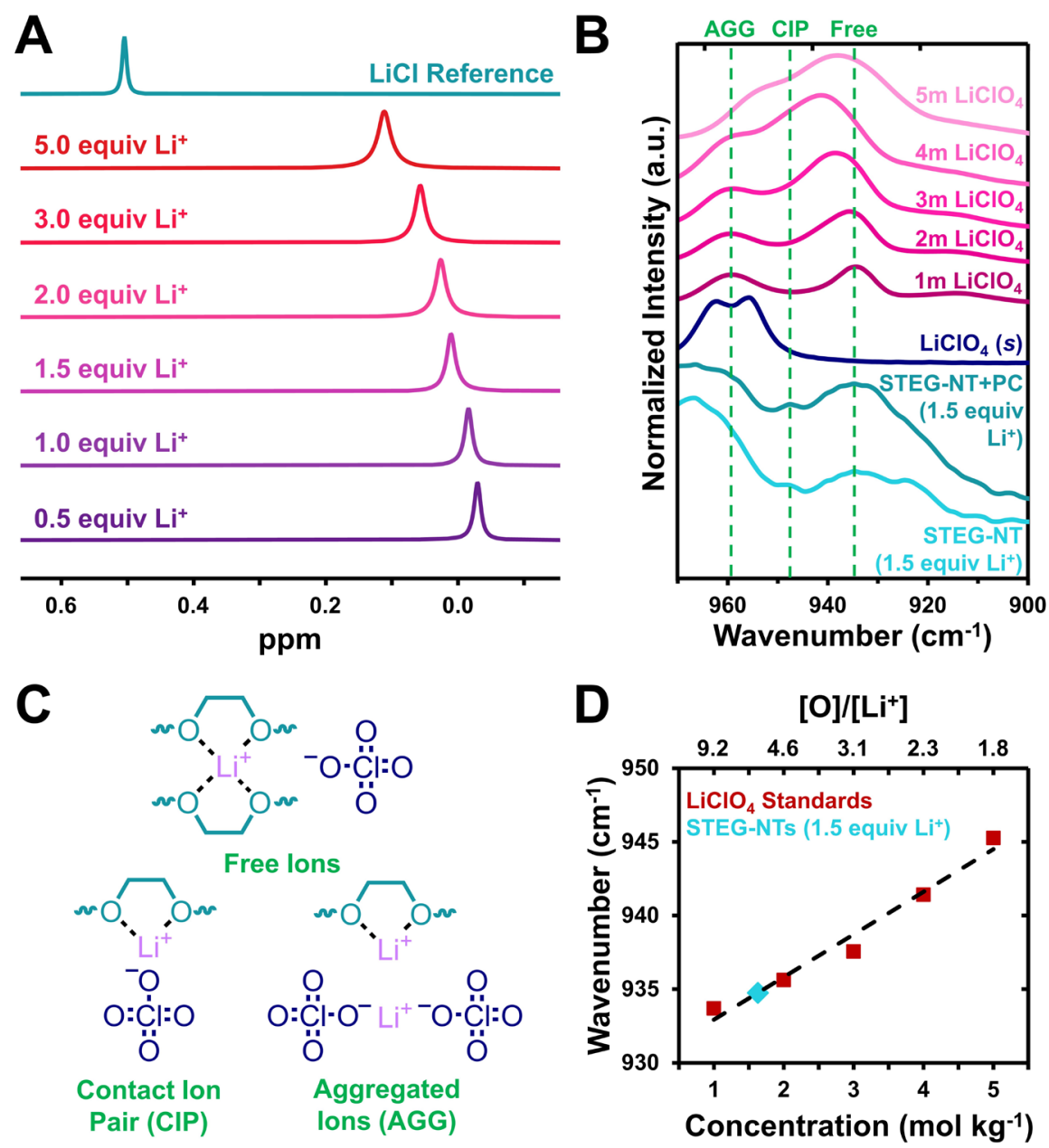

Figure 5. ${ }^{7} \mathrm{Li}$ NMR and Raman investigations into the ion solvation structures within STEG-NTs. (A) ${ }^{7} \mathrm{Li}$ NMR spectra of STEG-NTs treated with varying amounts of $\mathrm{LiCl}$ and a $\mathrm{LiCl}$ reference solution taken in the absence of coordinating groups. (B) Raman spectra of STEG-NTs lithiated with 1.5 equiv of $\mathrm{LiClO}_{4}$ before and after adding the $\mathrm{PC}$ plasticizer, along with $\mathrm{LiClO}_{4}$ standard solutions of varying concentration in PC. (C) Schematic of potential ion solvation structures within STEG-NTs. (D) Linear regression of the $\mathrm{Cl}-\mathrm{O}$ symmetric stretching peak position in the Raman spectra of $\mathrm{LiClO}_{4}$ standard solutions in $\mathrm{PC}$. The peak shift of STEG-NTs that had been lithiated with 1.5 equiv of $\mathrm{LiClO}_{4}$ was also marked to interpolate the $[\mathrm{O}] /\left[\mathrm{Li}^{+}\right]$ratio in the nanotube interior. 
Comparison of the conductivity of STEG-NTs with an unfunctionalized nanotube implicates the confined glycol chains as the source of efficient Li-ion conduction. DAPP was condensed with isophthalaldehyde to yield a macrocycle and nanotube lacking interior glycol functionality (IDA-NT, Figure 6A). IDA-NTs were lithiated with 1.5 equiv of $\mathrm{Li}^{+}$per macrocycle, pelletized, and subjected to EIS measurements. Compared to the glycol functionalized system, IDA-NTs exhibited an order of magnitude decrease in their conductivity $\left(3.10 \times 10^{-6} \mathrm{~S} \mathrm{~cm}^{-1} @ 30^{\circ} \mathrm{C}\right)$ (Figures 6B, 6C, and S46). Similarly, variable temperature EIS yielded an increase in $E_{\mathrm{a}}(0.59 \mathrm{eV})$ when compared to STEG-NTs (Figures 6B and S46). The increase in activation energy and decrease in conductivity when compared to STEG-NTs suggests that the presence of glycol moieties within the nanotube pore facilitate efficient Li-ion conduction. Furthermore, this comparison validates that structural regularity alone is insufficient to promote ion conduction, thereby validating the importance of nanotube pore functionalization methods for tuning emergent ionic conductivity.

In the absence of acid STEG-MCs show inferior ionic conductivity and undesired solubility when compared to STEG-NTs. Lithiating neutral STEG-MCs with 1.5 equiv of $\mathrm{Li}^{+}$per macrocycle resulted in a material with a room temperature conductivity lower than that of STEG-NTs $\left(2.43 \times 10^{-5} \mathrm{~S} \mathrm{~cm}^{-1}\right.$, Figure S45). Variable temperature EIS measurements demonstrate that pellets of neutral STEG-MCs conduct $\mathrm{Li}^{+}$to some degree $\left(E_{\mathrm{a}}=0.38 \mathrm{eV}\right.$, Figures $6 \mathrm{~B}, 6 \mathrm{C}$, and $\left.\mathrm{S} 45\right)$. We attribute the higher-than-expected conductivity of STEG-MCs to a degree of assembly occurring in the solid state driven by the amphiphilicity of the macrocycle (Figure S29). Despite forming a crystalline material in the solid state, molecularly dissolved STEG-MC showed no observable diffraction features (Figure S29). Due to a lack of order in solution, STEG-MCs are not amenable to many solution processing techniques, as casting films of STEG-MC would result in disordered, poorly conductive materials. These observations highlight an immediate advantage of STEG-NTs, in that the acid-mediated assembly occurs in solution, thereby enabling the solution casting of highly conductive films into device relevant morphologies. In addition to solubility plaguing their practical relevance, neutral STEG-MCs exhibit poor conductivity at elevated temperatures (Figure S49). Taken together, these observations underscore the need for robust noncovalent interactions, which are present in the high-aspect ratio STEG-NTs, as weaker non-covalent interactions result in poorly processable materials that lack thermomechanical stability. 

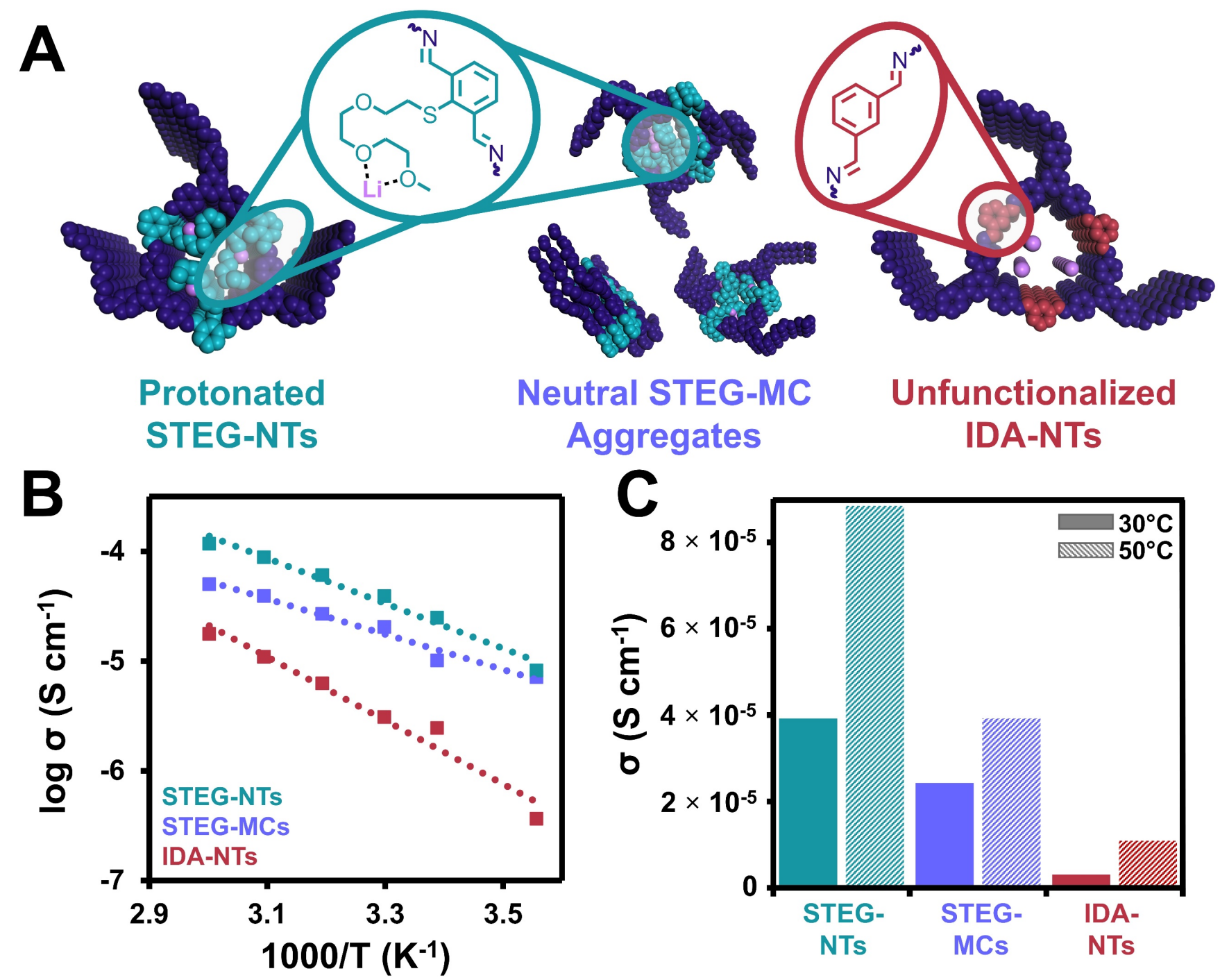

Figure 6. Li-ion conductivity performance of STEG-NTs compared to two control systems which either lack glycol functionalities or substantial 1D order. (A) Schematic of nanostructures analyzed. (B) Arrhenius plots of STEG-NTs, STEG-MC aggregates, and IDA-NTs loaded with 1.5 equiv of $\mathrm{Li}^{+}$. (C) Comparison of the conductivity of the three systems at $30^{\circ} \mathrm{C}$ (solid) and $50{ }^{\circ} \mathrm{C}$ (dashed), showing the poor performance of STEG-MC aggregates at elevated temperatures.

The suitability of STEG-NTs in a battery cell was investigated by performing linear sweep voltammetry (LSV) and Li plating/stripping experiments. LSV was used to assess the electrochemical stability of STEG-NTs within the traditional Li-ion battery operating window (-1 to $6 \mathrm{~V}$ vs $\left.\mathrm{Li} / \mathrm{Li}^{+}\right)$. A STEG-NT pellet was sandwiched between Li-metal and stainless-steel foil and scanned at a rate of $1 \mathrm{mV} \mathrm{s}^{-1}$ at 50 ${ }^{\circ}$ C. STEG-NTs exhibit higher oxidative stability when compared to traditional PEO-based polymer electrolytes (Figure 7A), ${ }^{50-54}$ which is attributed to the confinement of the glycol moieties within the nanotube interior, thereby shielding them from undesired oxidation. The oxidative stability was preserved when tested in the presence of a carbon conductive agent, proving that the low electronic conductivity of 
the polymer electrolyte is not responsible for the observed oxidative stability (Figure S51). STEG-NTs are amenable to reversible $\mathrm{Li}$ deposition $\left(-1 \mathrm{~V}\right.$ vs $\left.\mathrm{Li} / \mathrm{Li}^{+}\right)$and stripping $\left(0.6 \mathrm{~V}\right.$ vs $\left.\mathrm{Li} / \mathrm{Li}^{+}\right)$, implying their structural and electrochemical stability in the potential LIB anode range (Figure 7B). The electrochemical feature at $0.7 \mathrm{~V}\left(\mathrm{vs} \mathrm{Li} / \mathrm{Li}^{+}\right)$is due to the formation of a solid electrolyte interphase (SEI layer). Cycling $\mathrm{Li}$ plating-stripping experiments were carried out to assess the long-term stability of STEG-NTs towards standard lithium-ion battery conditions. A Li/Li symmetric cell was prepared by pelletizing STEG-NTs between two Li-metal electrodes. Galvanostatic Li plating-stripping on the Li-metal electrodes was repeatedly conducted at a current density of $0.01 \mathrm{~mA} \mathrm{~cm}^{-2}$ for $2 \mathrm{~h}$ per cycle. Over the course of $500 \mathrm{~h}$, STEG-NTs demonstrated reversible stripping and plating processes without fingerprints of material degradation (Figures 7C and 7D). STEG-NTs also exhibited the ability to reversibly plate and strip Li at varying current densities (Figure S53). Cross-sectional SEM images of the Li-metal electrode demonstrated that Li plating and stripping occurred uniformly at the interface between the electrodes and the nanotube electrolyte layer (Figures S54-55). The smooth interface of the Li-metal electrode after cycling indicates that STEG-NTs promote uniform Li-ion flux to and from the metal electrode. The bright band uniformly formed at the interface indicates the formation of a stable SEI layer. Together, these results emphasize the potential of STEG-NTs towards practical Li-ion battery cells, and more broadly show the promise of these macrocycle assemblies under application relevant conditions. 

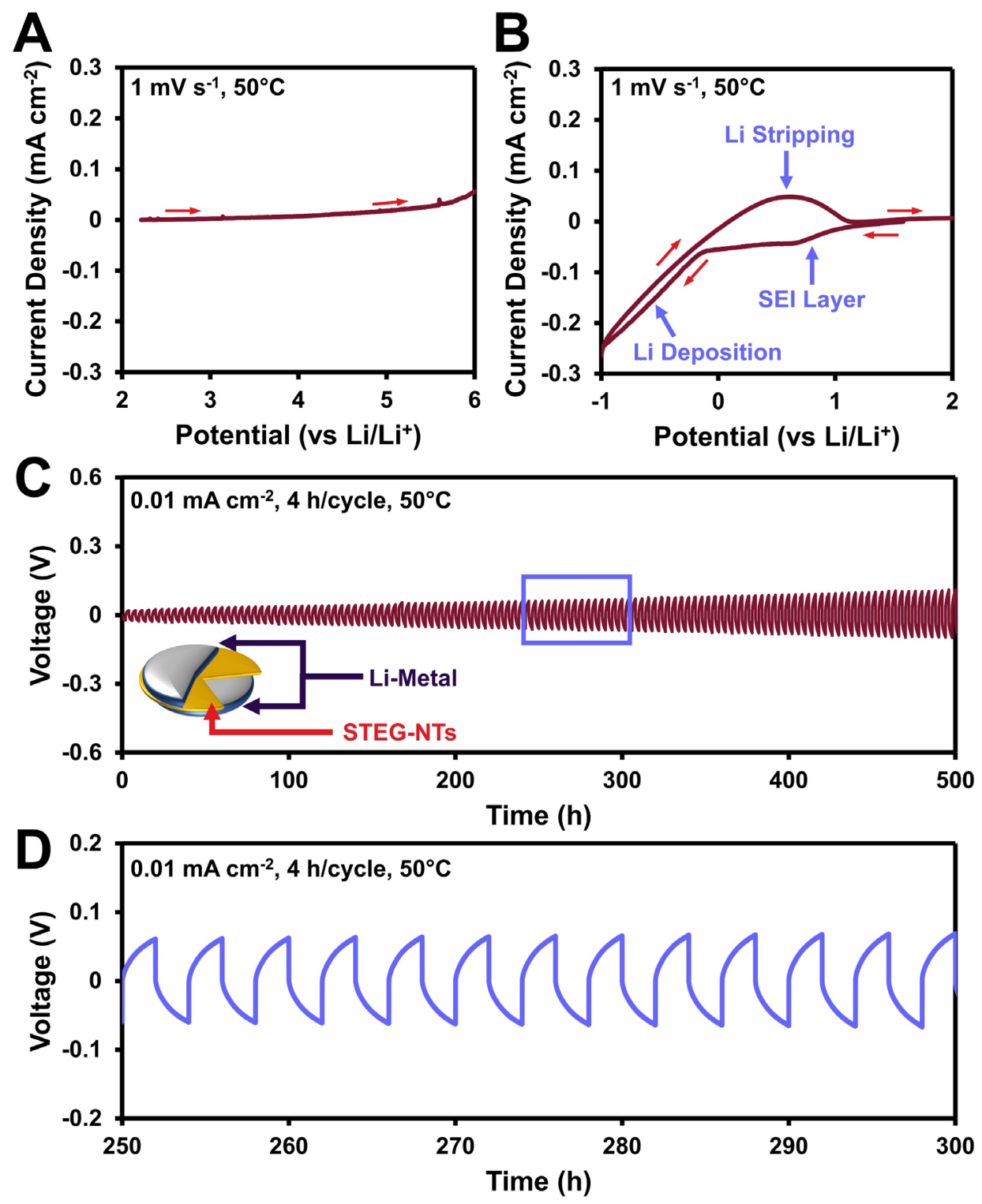

Figure 7. Application of STEG-NTs to practical Li-ion battery cells. LSV curves upon (A) oxidation and (B) reduction of STEG-NT pellets. (C) Galvanostatic Li plating-stripping cycles of STEG-NTs when pelletized between two Li-metal electrodes. (D) Magnified view of the Li plating-stripping test between 250 and $300 \mathrm{~h}$, highlighted by the purple box in $5 \mathrm{C}$.

\section{Conclusions}

We have designed a mechanically robust and solution processable nanotube with three interior triethylene glycol moieties for emergent $\mathrm{Li}^{+}$ion conduction. Condensation of DAPP with STEG-IDA yields an amphiphilic macrocycle in high isolated yield. Protonation of the pyridine moieties within DAPP under the mild macrocycle synthesis conditions drives the formation of high-aspect ratio nanotubes with three interior triethylene glycol moieties (STEG-NTs). In Solvo XRD measurements demonstrated both nanotube formation and pore-functionalization as evident by diffraction features at 0.17 and $0.2 \AA^{-1}$, respectively. AFM and SEM imaging confirmed that mesoscale nanotube assemblies are formed with 
lengths exceeding $10 \mu \mathrm{m}$. Upon lithiating STEG-NTs with 1.5 equiv of $\mathrm{Li}^{+}$per macrocycle, a system with substantial Li-ion conductivity of $3.91 \times 10^{-5} \mathrm{~S} \mathrm{~cm}^{-1}$ at room temperature and a low activation energy $(0.42$ eV) was realized. ${ }^{7} \mathrm{Li}$ NMR and Raman spectroscopy demonstrated that lithiation exclusively took place within the nanotube interior, and that the glycol groups are directly involved in $\mathrm{Li}^{+}$transduction. Comparing the conductivities of the amphiphilic nanotubes with IDA-NTs and weakly supported STEGMC aggregates, it was demonstrated that both glycol moieties and robust non-covalent interactions are required to realize a highly conductive material. LSV and Li plating-stripping tests demonstrated that STEG-NTs are stable under device relevant conditions for over 500 hours of operation, highlighting the potential of this materials platform in the construction of practical ion-conduction materials. Overall, these

findings highlight the potential of precisely functionalized synthetic nanotubes in ion conduction applications and will inspire future studies into optimizing performance through coupled molecular and supramolecular design.

\section{ASSOCIATED CONTENT}

Supporting Information. The Supporting Information is available free of charge on the ChemRxiv Server:

Instrumentation, synthetic and experimental procedures, NMR spectra, FTIR spectra, GPC chromatograms, MALDI-MS spectra, in solvo XRD patterns, Pawley structural refinements, powder XRD patterns, SEM images, AFM images, EIS methods and data, LSV curves, Li plating-stripping test results.

\section{AUTHOR INFORMATION}

\section{Corresponding Authors}

*JWC: jangwookchoi@snu.ac.kr

*WRD: wdichtel@northwestern.edu

\section{ORCID}

Michael J. Strauss: 0000-0003-0808-2568

Insu Hwang: 0000-0002-3642-6869

Austin M. Evans: 0000-0002-3597-2454

Anusree Natraj: 0000-0003-4171-9815

Xavier Aguilar-Enriquez: 0000-0002-5940-4427

Ioannina Castano: 0000-0001-6491-2170

Emily K. Roesner: 0000-0003-0583-7371

Jang Wook Choi: 0000-0001-8783-0901

William R. Dichtel: 0000-0002-3635-6119 


\section{Notes}

The authors declare no competing financial interests.

\section{Acknowledgements}

This work was funded by the Army Research Office through the Multidisciplinary University Research Initiative (MURI; W911NF-15-1-04477, to W.R.D.). M.J.S was supported by the National Science Foundation (NSF) through the Graduate Research Fellowship Program (GRFP) under Grant No. (DGE1842165). M.J.S. was partially supported by the Ryan Fellowship and the International Institute for Nanotechnology. A.M.E. was supported by the NSF through the GRFP under Grant No. (DGE-1324585). I.C. was supported by the NSF through the GRFP under Grant No. (DGE-1842165). X.A. was supported by the NSF through the GRFP under Grant No. (DGE-1842165). This work made use of the Integrated Molecular Structure Education and Research Center (IMSERC) at Northwestern University, which has received support from the NSF (CHE-1048773), the Soft and Hybrid Nanotechnology Experimental (SHyNE) Resource (NSF; NNCI-1542205), the State of Illinois, and the International Institute for Nanotechnology (IIN). This work also made use of the Scanned Probe Imaging and Development (SPID), and the Electron Probe Instrumentation Center (EPIC), facilities of Northwestern University's Atomic and Nanoscale Characterization Experiment Center (NUANCE), which has received support from the Soft and Hybrid Nanotechnology Experimental (SHyNE) Resource (NSF; ECCS-1542205); the MRSEC program (NSF; DMR-1720139) at the Materials Research Center; the International Institute for Nanotechnology (IIN); the Keck Foundation; and the State of Illinois. Instruments used in this work were also supported by the Northwestern University Keck Biophysics Facility and a Cancer Center Support Grant (NCI CA060553). Parts of this work were performed at the DuPont-Northwestern-Dow Collaborative Access Team (DND-CAT) located at Sector 5 of the Advanced Photon Source (APS) at Argonne National Lab. This research used resources of the Advanced Photon Source and the Center for Nanoscale Materials, both U.S. Department of Energy (DOE) Office of Science User Facilities operated for the DOE Office of Science by Argonne National Laboratory under Grant No. (DGE-1324585). J.W.C. acknowledges financial support from a National Research Foundation of Korea grant (NRF2017M1A2A2044504 and NRF-2021R1A2B5B03001956) and generous support from Institute of Engineering Research (IOER) and Inter-university Semiconductor Research Center (ISRC) at Seoul National University. We acknowledge Prof. Julia Kalow for the use of her GPC instrument.

\section{References}

1. Gestel, J. v.; Schoot, P. v. d.; Michels, M. A. J., Amplification of Chirality in Helical Supramolecular Polymers Beyond the Long-Chain Limit. J. Chem. Phys. 2004, 120, 8253-8261. 
2. Yashima, E.; Ousaka, N.; Taura, D.; Shimomura, K.; Ikai, T.; Maeda, K., Supramolecular Helical Systems: Helical Assemblies of Small Molecules, Foldamers, and Polymers with Chiral Amplification and Their Functions. Chem. Rev. 2016, 116, 13752-13990.

3. Eisele, D. M.; Arias, D. H.; Fu, X.; Bloemsma, E. A.; Steiner, C. P.; Jensen, R. A.; Rebentrost, P.; Eisele, H.; Tokmakoff, A.; Lloyd, S.; Nelson, K. A.; Nicastro, D.; Knoester, J.; Bawendi, M. G., Robust Excitons Inhabit Soft Supramolecular Nanotubes. Proc. Natl. Acad. Sci. U.S.A. 2014, 111, E3367-E3375. 4. Wagner, W.; Wehner, M.; Stepanenko, V.; Ogi, S.; Würthner, F., Living Supramolecular Polymerization of a Perylene Bisimide Dye into Fluorescent J-Aggregates. Angew. Chem. Int. Ed. 2017, 56, 16008-16012.

5. Brixner, T.; Hildner, R.; Köhler, J.; Lambert, C.; Würthner, F., Exciton Transport in Molecular Aggregates - From Natural Antennas to Synthetic Chromophore Systems. Adv. Energy Mater. 2017, 7, 1700236.

6. Li, C.; Iscen, A.; Sai, H.; Sato, K.; Sather, N. A.; Chin, S. M.; Álvarez, Z.; Palmer, L. C.; Schatz, G. C.; Stupp, S. I., Supramolecular-Covalent Hybrid Polymers for Light-Activated Mechanical Actuation. Nat. Mater. 2020, 19, 900-909.

7. Cate, t. A. T.; Beek, v. D. J. M.; Spiering, A. J. H.; Dankers, P. Y. W.; Sijbesma, R. P.; Meijer, E. W., Hydrogen-Bonded Supramolecular Polymers with Tunable Material Properties. Polymer Preprints 2003, 44, $618-619$.

8. Bosman, A. W.; Sijbesma, R. P.; Meijer, E. W., Supramolecular Polymers At Work. Mater. Today 2004, 7, 34-39.

9. Greenfield, M. A.; Hoffman, J. R.; Olvera de la Cruz, M.; Stupp, S. I., Tunable Mechanics of Peptide Nanofiber Gels. Langmuir 2010, 26, 3641-3647.

10. van Rossum, S. A. P.; Tena-Solsona, M.; van Esch, J. H.; Eelkema, R.; Boekhoven, J., Dissipative out-of-equilibrium assembly of man-made supramolecular materials. Chem. Soc. Rev. 2017, 46, 55195535.

11. Wanzke, C.; Jussupow, A.; Kohler, F.; Dietz, H.; Kaila, V. R. I.; Boekhoven, J., Dynamic Vesicles Formed By Dissipative Self-Assembly. ChemSystemsChem 2020, 2, e1900044.

12. Dai, K.; Fores, J. R.; Wanzke, C.; Winkeljann, B.; Bergmann, A. M.; Lieleg, O.; Boekhoven, J., Regulating Chemically Fueled Peptide Assemblies by Molecular Design. J. Am. Chem. Soc. 2020, 142, 14142-14149.

13. Kriebisch, B. A. K.; Jussupow, A.; Bergmann, A. M.; Kohler, F.; Dietz, H.; Kaila, V. R. I.; Boekhoven, J., Reciprocal Coupling in Chemically Fueled Assembly: A Reaction Cycle Regulates Self-Assembly and Vice Versa. J. Am. Chem. Soc. 2020, 142, 20837-20844.

14. Sun, C.; Shen, M.; Chavez, A. D.; Evans, A. M.; Liu, X.; Harutyunyan, B.; Flanders, N. C.; Hersam, M. C.; Bedzyk, M. J.; Olvera de la Cruz, M.; Dichtel, W. R., High Aspect Ratio Nanotubes Assembled From Macrocyclic Iminium Salts. Proc. Natl. Acad. Sci. U.S.A. 2018, 115, 8883-8888.

15. Strauss, M. J.; Asheghali, D.; Evans, A. M.; Li, R. L.; Chavez, A. D.; Sun, C.; Becker, M. L.; Dichtel, W. R., Cooperative Self-Assembly of Pyridine-2,6-Diimine-Linked Macrocycles into Mechanically Robust Nanotubes. Angew. Chem. Int. Ed. 2019, 58, 14708-14714.

16. Strauss, M. J.; Evans, A. M.; Castano, I.; Li, R. L.; Dichtel, W. R., Supramolecular Polymerization Provides Non-Equilibrium Product Distributions of Imine-Linked Macrocycles. Chem. Sci. 2020, 11, 1957-1963.

17. Strauss, M. J.; Jia, M.; Evans, Austin M.; Castano, I.; Li, R. L.; Aguilar-Enriquez, X.; Roesner, E. K.; Swartz, J. L.; Chavez, A. D.; Enciso, A. E.; Stoddart, J. F.; Rolandi, M.; Dichtel, W. R., Diverse ProtonConducting Nanotubes via a Tandem Macrocyclization and Assembly Strategy. ChemRxiv 2021.

18. Mackanic, D. G.; Yan, X.; Zhang, Q.; Matsuhisa, N.; Yu, Z.; Jiang, Y.; Manika, T.; Lopez, J.; Yan, H.; Liu, K.; Chen, X.; Cui, Y.; Bao, Z., Decoupling of Mechanical Properties and Ionic Conductivity in Supramolecular Lithium Ion Conductors. Nat. Commun. 2019, 10, 5384. 
19. Manthiram, A.; Yu, X.; Wang, S., Lithium Battery Chemistries Enabled by Solid-State Electrolytes. Nat. Rev. Mater. 2017, 2, 16103.

20. Zhou, D.; Shanmukaraj, D.; Tkacheva, A.; Armand, M.; Wang, G., Polymer Electrolytes for LithiumBased Batteries: Advances and Prospects. Chem 2019, 5, 2326-2352.

21. Kraytsberg, A.; Ein-Eli, Y., Review of Advanced Materials for Proton Exchange Membrane Fuel Cells. Energy \& Fuels 2014, 28, 7303-7330.

22. Borup, R.; Meyers, J.; Pivovar, B.; Kim, Y. S.; Mukundan, R.; Garland, N.; Myers, D.; Wilson, M.; Garzon, F.; Wood, D.; Zelenay, P.; More, K.; Stroh, K.; Zawodzinski, T.; Boncella, J.; McGrath, J. E.; Inaba, M.; Miyatake, K.; Hori, M.; Ota, K.; Ogumi, Z.; Miyata, S.; Nishikata, A.; Siroma, Z.; Uchimoto, Y.; Yasuda, K.; Kimijima, K.-i.; Iwashita, N., Scientific Aspects of Polymer Electrolyte Fuel Cell Durability and Degradation. Chem. Rev. 2007, 107, 3904-3951.

23. Vilela, C.; Silvestre, A. J. D.; Figueiredo, F. M. L.; Freire, C. S. R., Nanocellulose-Based Materials as Components of Polymer Electrolyte Fuel Cells. J. Mater. Chem. A. 2019, 7, 20045-20074.

24. Zhang, Y.; Duan, J.; Ma, D.; Li, P.; Li, S.; Li, H.; Zhou, J.; Ma, X.; Feng, X.; Wang, B., ThreeDimensional Anionic Cyclodextrin-Based Covalent Organic Frameworks. Angew. Chem. Int. Ed. 2017, 56, 16313-16317.

25. Guo, Z.; Zhang, Y.; Dong, Y.; Li, J.; Li, S.; Shao, P.; Feng, X.; Wang, B., Fast Ion Transport Pathway Provided by Polyethylene Glycol Confined in Covalent Organic Frameworks. J. Am. Chem. Soc. 2019, 141, 1923-1927.

26. Jadhav, T.; Fang, Y.; Liu, C.-H.; Dadvand, A.; Hamzehpoor, E.; Patterson, W.; Jonderian, A.; Stein, R. S.; Perepichka, D. F., Transformation between 2D and 3D Covalent Organic Frameworks via Reversible [2+2] Cycloaddition. J. Am. Chem. Soc. 2020, 142, 8862-8870.

27. Burke, D. W.; Sun, C.; Castano, I.; Flanders, N. C.; Evans, A. M.; Vitaku, E.; McLeod, D. C.; Lambeth, R. H.; Chen, L. X.; Gianneschi, N. C.; Dichtel, W. R., Acid Exfoliation of Imine-linked Covalent Organic Frameworks Enables Solution Processing into Crystalline Thin Films. Angew. Chem. Int. Ed. 2020, 59, 5165-5171.

28. Fischbach, D. M.; Rhoades, G.; Espy, C.; Goldberg, F.; Smith, B. J., Controlling the crystalline structure of imine-linked 3D covalent organic frameworks. Chem. Commun. 2019, 55, 3594-3597.

29. Rodríguez-San-Miguel, D.; Zamora, F., Processing of Covalent Organic Frameworks: An Ingredient For a Material to Succeed. Chem. Soc. Rev. 2019, 48, 4375-4386.

30. Xu, Q.; Tao, S.; Jiang, Q.; Jiang, D., Ion Conduction in Polyelectrolyte Covalent Organic Frameworks. J. Am. Chem. Soc. 2018, 140, 7429-7432.

31. Chen, H.; Tu, H.; Hu, C.; Liu, Y.; Dong, D.; Sun, Y.; Dai, Y.; Wang, S.; Qian, H.; Lin, Z.; Chen, L., Cationic Covalent Organic Framework Nanosheets for Fast Li-Ion Conduction. J. Am. Chem. Soc. 2018, 140, 896-899.

32. Kříž, J.; Abbrent, S.; Dybal, J.; Kurková, D.; Lindgren, J.; Tegenfeldt, J.; Wendsjö, Å., Nature and Dynamics of Lithium Ion Coordination in Oligo(ethylene glycol) Dimethacrylate-Solvent Systems: NMR, Raman, and Quantum Mechanical Study. J. Phys. Chem A. 1999, 103, 8505-8515.

33. Kao, H.-M.; Chang, P.-C.; Chao, S.-W.; Lee, C.-H., ${ }^{7}$ Li NMR, Ionic Conductivity and Self-Diffusion Coefficients of Lithium Ion and Solvent of Plasticized Organic-Inorganic Hybrid Electrolyte Based on PPG-PEG-PPG Diamine and Alkoxysilanes. Electrochim. Acta 2006, 52, 1015-1027.

34. Asano, A.; Takegoshi, K.; Hikichi, K., ${ }^{13} \mathrm{C} \mathrm{CP} / \mathrm{MAS}$ and ${ }^{7} \mathrm{Li}$ NMR Study of Lithium Perchlorate/Poly(ethylene oxide). Polym. J. 1999, 31, 602-608.

35. Reddy, M. J.; Chu, P. P., ${ }^{7} \mathrm{Li}$ NMR Spectroscopy and Ion Conduction Mechanism in Mesoporous Silica (SBA-15) Composite Poly(ethylene oxide) Electrolyte. J. Power Sources 2004, 135, 1-8.

36. Bernson, A.; Lindgren, J.; Huang, W.; Frech, R., Coordination and conformation in PEO, PEGM and PEG Systems ContainingLithium or LanthanumTriflate. Polymer 1995, 36, 4471-4478. 
37. Kamiyama, Y.; Shibata, M.; Kanzaki, R.; Fujii, K., Lithium-Ion Coordination-Induced Conformational Change of PEG Chains in Ionic-Liquid-Based Electrolytes. Phys. Chem. Chem. Phys. 2020, 22, 5561-5567.

38. Shah, F. U.; Gnezdilov, O. I.; Gusain, R.; Filippov, A., Transport and Association of Ions in Lithium Battery Electrolytes Based on Glycol Ether Mixed with Halogen-Free Orthoborate Ionic Liquid. Sci. Rep. 2017, 7, 16340.

39. Chavez, A. D.; Evans, A. M.; Flanders, N. C.; Bisbey, R. P.; Vitaku, E.; Chen, L. X.; Dichtel, W. R., Equilibration of Imine-Linked Polymers to Hexagonal Macrocycles Driven by Self-Assembly. Chem. Eur. J. 2018, 24, 3989-3993.

40. Zhai, H.; Xu, P.; Ning, M.; Cheng, Q.; Mandal, J.; Yang, Y., A Flexible Solid Composite Electrolyte with Vertically Aligned and Connected Ion-Conducting Nanoparticles for Lithium Batteries. Nano Lett. 2017, 17, 3182-3187.

41. Son, C. Y.; Wang, Z.-G., Ion Transport in Small-Molecule and Polymer Electrolytes. J. Chem. Phys. 2020, 153, 100903.

42. Patra, S.; Thakur, P.; Soman, B.; Puthirath, A. B.; Ajayan, P. M.; Mogurampelly, S.; Karthik Chethan, V.; Narayanan, T. N., Mechanistic Insight Into the Improved Li Ion Conductivity of Solid Polymer Electrolytes. RSC Adv. 2019, 9, 38646-38657.

43. Robitaille, C. D.; Fauteux, D., Phase Diagrams and Conductivity Characterization of Some PEO LiX Electrolytes. J. Electrochem. Soc. 1986, 133, 315-325.

44. Cheng, S.; Smith, D. M.; Pan, Q.; Wang, S.; Li, C. Y., Anisotropic Ion Transport in Nanostructured Solid Polymer Electrolytes. RSC Adv. 2015, 5, 48793-48810.

45. Huang, Y.-P.; Chow, T. J., Polymer Electrolyte Containing Dialkoxyacenes with Oligo(Ethylene Oxide) Side Chains. J. Chin. Chem. Soc. 2006, 53, 1335-1342.

46. Chabanel, M.; Legoff, D.; Touaj, K., Aggregation of perchlorates in aprotic donor solvents. Part 1.Lithium and sodium perchlorates. J. Chem. Soc., Faraday Trans. 1996, 92, 4199-4205.

47. James, D.; Mayes, R., Ion-ion-solvent interactions in solution. I. Solutions of $\mathrm{LiClO}_{4}$ in acetone. Aust. J. Chem. 1982, 35, 1775-1784.

48. Sagane, F.; Abe, T.; Ogumi, Z., Li+-Ion Transfer through the Interface between $\mathrm{Li}^{+}$-Ion Conductive Ceramic Electrolyte and $\mathrm{Li}^{+}$-Ion-Concentrated Propylene Carbonate Solution. J. Phys. Chem. C. 2009, 113, 20135-20138.

49. Hwang, S.; Kim, D.-H.; Shin, J. H.; Jang, J. E.; Ahn, K. H.; Lee, C.; Lee, H., Ionic Conduction and Solution Structure in $\mathrm{LiPF}_{6}$ and $\mathrm{LiBF}_{4}$ Propylene Carbonate Electrolytes. J. Phys. Chem C. 2018, 122, 19438-19446.

50. Nie, K.; Wang, X.; Qiu, J.; Wang, Y.; Yang, Q.; Xu, J.; Yu, X.; Li, H.; Huang, X.; Chen, L., Increasing Poly(ethylene oxide) Stability to 4.5 V by Surface Coating of the Cathode. ACS Energy Lett. 2020, 5, 826832.

51. Xia, Y.; Fujieda, T.; Tatsumi, K.; Prosini, P. P.; Sakai, T., Thermal and Electrochemical Stability of Cathode Materials in Solid Polymer Electrolyte. J. Power Sources 2001, 92, 234-243.

52. Wu, J.; Rao, Z.; Cheng, Z.; Yuan, L.; Li, Z.; Huang, Y., Ultrathin, Flexible Polymer Electrolyte for Cost-Effective Fabrication of All-Solid-State Lithium Metal Batteries. Adv. Energy Mater. 2019, 9, 1902767.

53. Wan, Z.; Lei, D.; Yang, W.; Liu, C.; Shi, K.; Hao, X.; Shen, L.; Lv, W.; Li, B.; Yang, Q.-H.; Kang, F.; He, Y.-B., Low Resistance-Integrated All-Solid-State Battery Achieved by $\mathrm{Li}_{7} \mathrm{La}_{3} \mathrm{Zr}_{2} \mathrm{O}_{12}$ Nanowire Upgrading Polyethylene Oxide (PEO) Composite Electrolyte and PEO Cathode Binder. Adv. Funct. Mater. 2019, 29, 1805301.

54. Li, Z.; Zhang, H.; Sun, X.; Yang, Y., Mitigating Interfacial Instability in Polymer Electrolyte-Based Solid-State Lithium Metal Batteries with 4 V Cathodes. ACS Energy Lett. 2020, 5, 3244-3253. 